\title{
Potential metabolic resistance mechanisms to ivermectin in Anopheles gambiae: a synergist bioassay study
}

\author{
Patricia Nicolas ${ }^{1,2+}$, Caroline Kiuru ${ }^{1,2+}$, Martin G. Wagah ${ }^{3,4}$, Martha Muturi ${ }^{4}$, Urs Duthaler ${ }^{5,6}$, Felix Hammann ${ }^{7}$, \\ Marta Maia ${ }^{4,8}$ and Carlos Chaccour ${ }^{1,9,10^{*}}$ (i)
}

\begin{abstract}
Background: Despite remarkable success obtained with current malaria vector control strategies in the last 15 years, additional innovative measures will be needed to achieve the ambitious goals for malaria control set for 2030 by the World Health Organization (WHO). New tools will need to address insecticide resistance and residual transmission as key challenges. Endectocides such as ivermectin are drugs that kill mosquitoes which feed on treated subjects. Mass administration of ivermectin can effectively target outdoor and early biting vectors, complementing the still effective conventional tools. Although this approach has garnered attention, development of ivermectin resistance is a potential pitfall. Herein, we evaluate the potential role of xenobiotic pumps and cytochrome P450 enzymes in protecting mosquitoes against ivermectin by active efflux and metabolic detoxification, respectively.
\end{abstract}

Methods: We determined the lethal concentration 50 for ivermectin in colonized Anopheles gambiae; then we used chemical inhibitors and inducers of xenobiotic pumps and cytochrome P450 enzymes in combination with ivermectin to probe the mechanism of ivermectin detoxification.

Results: Dual inhibition of xenobiotic pumps and cytochromes was found to have a synergistic effect with ivermectin, greatly increasing mosquito mortality. Inhibition of xenobiotic pumps alone had no effect on ivermectin-induced mortality. Induction of xenobiotic pumps and cytochromes may confer partial protection from ivermectin.

Conclusion: There is a clear pathway for development of ivermectin resistance in malaria vectors. Detoxification mechanisms mediated by cytochrome P450 enzymes are more important than xenobiotic pumps in protecting mosquitoes against ivermectin.

Keywords: Ivermectin, Endectocide, Resistance, Insecticide resistance, CYP, P-gp, ABC transporter, Synergists, Bioassay

\section{Background}

Since the turn of the century, significant advances have been made against malaria; the global malaria mortality

\footnotetext{
*Correspondence: carlos.chaccour@isglobal.org

'Patricia Nicolas and Caroline Kiuru contributed equally to this work

1 ISGlobal, Hospital Clínic-Universitat de Barcelona, Rosello 132, 5a 2a 08036 Barcelona, Spain

Full list of author information is available at the end of the article
}

rate has reduced by more than $50 \%$, saving more than 6.8 million lives $[1,2]$. Two vector control measures are mainly responsible for this success, (1) the use of insecticide-treated nets and (2) indoor residual spraying, both of which are insecticide-based and home-centered [3]. The continuous use and reliance on insecticides has put selective pressure on the mosquitoes, radically changing the vector species' distribution and behavior [4]. This allows malaria transmission to continue by shifting 
to times and spaces unprotected by the current vector control measures, most noticeably early biting and/or outdoor biting [5]. Moreover, the selection pressure has yielded mosquito populations that are resistant to the current insecticides used in malaria vector control [6].

The new challenges in vector control urgently call for the development of new tools to circumvent them [7]. One of the proposed tools currently under evaluation is the use of endectocides [8]. Endectocides are antiparasitic drugs with activity against endoparasites and ectoparasites such as mosquitoes which feed on treated humans or animals. Importantly, endectocides can target exophilic and exophagic vectors, tackling the problem of residual transmission, while perfectly complementing the indoor vector control measures [9].

Owing to its excellent safety profile and activity against most malaria vectors, ivermectin is the leading endectocide candidate for malaria control [10,11]. In addition, ivermectin is used extensively for the control of neglected tropical diseases (NTDs) that overlap with malaria in endemic areas, potentially increasing the cost-effectiveness of implementing ivermectin mass drug administration (MDA) [12, 13].

Several of the effects ivermectin has on malaria vectors point towards a low risk and slow speed for the development of resistance. These include (1) its mechanism of action, agonizing glutamate-gated chloride channels, which differs from all currently approved public health insecticides [14]; (2) direct delivery to the midgut with a blood meal that can bypass resistance associated with cuticular mechanisms [15]; and (3) marked reduction in fertility and fecundity of malaria vectors exposed to sublethal concentrations $[16,17]$. Although the development of ivermectin resistance in malaria vectors may take a long time, it is inevitable, as it has already been reported in other arthropods [18]. Moreover, considering that ivermectin MDA for NTDs has been ongoing for the past 30 years, these could have potentially exposed malaria vectors to mostly sublethal ivermectin concentrations that could enhance the process of resistance development [19]. Therefore, it is important to have an early and thorough understanding of potential mosquito detoxification mechanisms for ivermectin. This will be crucial for early development of approaches that could delay, counter, or detect ivermectin resistance.

Herein, we evaluated the potential detoxification mechanisms involved in the response to ivermectin in the Anopheles gambiae s.s. mosquito. We were mainly interested in two general mechanisms of detoxification: (1) metabolic detoxification mediated by enzymes, specifically cytochrome P450 enzymes (CYPs), and (2) detoxification by excretion facilitated by ATP binding cassette $(\mathrm{ABC})$ transporters, specifically P-glycoprotein (P-gp).
The increased activity of CYPs leads to increased biodegradation of the toxins, while increased activity of P-gp leads to increased excretion of xenobiotics. Both mechanisms reduce the toxic effects of the compounds by decreasing the insects' systemic exposure to them [20]. Both detoxification mechanisms have been implicated in resistance to insecticides used for the control of malaria vectors, with metabolic detoxification being the most common mechanism [21, 22]. Elevated levels of CYPs, esterases, and glutathione S-transferases (GSTs) are associated with resistance to different classes of insecticides [22]. Though both mechanisms are known to contribute to ivermectin resistance in other arthropods, their potential contribution to ivermectin resistance in mosquitoes has not been thoroughly explored [23-25].

In this study, we assessed the interaction of different chemical inhibitors and inducers of CYP and P-gp with ivermectin in An. gambiae s.s. (Kilifi strain) mosquitoes. Our main questions were whether and how the inhibitors and inducers of CYP and P-gp affect ivermectin-induced mosquito mortality.

\section{Methods \\ Experimental design}

The experiments were conducted in two phases. In phase 1 , an ivermectin dose-finding experiment was performed using triplicate batches of 50 female mosquitoes (3-5 days old) with the aim of identifying the 10 -day $\mathrm{LC}_{50}$ of ivermectin for our colony (Fig. 1). The 10-day period was chosen based on the minimum extrinsic incubation period of Plasmodium falciparum parasites, i.e. mosquitoes dying before 10 days are unlikely to become infectious [26]. We tested five different concentrations of ivermectin spanning $\pm 20-40 \%$ of the 5-day $\mathrm{LC}_{50}$ described by Kobylinski et al. [27], namely $4 \mathrm{ng} / \mathrm{ml}, 8 \mathrm{ng} / \mathrm{ml}, 12 \mathrm{ng} / \mathrm{ml}, 16 \mathrm{ng} / \mathrm{ml}$, and $20 \mathrm{ng} / \mathrm{ml}$.

In phase 2, following the identification of a concentration of ivermectin yielding about $50 \%$ mortality in 10 days, we evaluated (1) the effect on mosquito mortality of CYP and/or P-gp inhibitors and inducers alone at different concentrations and (2) the effect of combining ivermectin with different doses of CYP and/or P-gp inhibitors and inducers (Fig. 2).

For voriconazole, ritonavir, cobicistat, cyclosporine A, elacridar, and rifampicin, the concentrations tested were based on the maximum blood concentration reached in humans after a single dose $\left(C_{\max }\right)$ as reported in the literature (Table 1). Four concentrations corresponding to $C_{\max }, 75 \% C_{\max }, 50 \% C_{\max }$ and $25 \%$ $C_{\max }$ were evaluated. Hereafter, $C_{\max }$ concentration is referred to as A, $75 \% C_{\max }$ as $\mathrm{B}, 50 \% C_{\max }$ as $\mathrm{C}$, and $25 \%$ $C_{\text {max }}$ as D. 
Given that mosquitoes were to be exposed in batches to the different drugs and combinations, the study was considered cluster-randomized, in which the batch exposed to any drug was the unit of randomization and the mosquito was the analysis unit. The sample size was adjusted for a cluster effect. A 50\% increase in ivermectin-driven 10-day mosquito mortality was considered of potential public health value. According to the method reported by Hayes and Bennett [28], using three replicas of 50 mosquitoes per group gives the study $80 \%$ power at a $5 \%$ significance level to detect a $50 \%$ increase in 10 -day mortality from 50 to $75 \%$ by adding the synergist. This uses an intra-cluster correlation coefficient of 0.06 described before for mosquito colonies [29]. These calculations are confirmed using the formula of Gangnon and Kosorok [30], which shows a design effect of 3.34 , with $70 \%$ possibility of observing mortality within 10 days.

\section{Mosquitoes}

Throughout the study we used an Anopheles gambiae s.s. Kilifi strain maintained in KEMRI-Wellcome Trust Research Programme insectary in Kilifi, Kenya. The colony was adapted to the insectary in 2011 from larvae collected in Mbogolo, Kilifi county. The colony is fully insecticide-susceptible and is subjected to quarterly resistance monitoring using WHO tube tests.

The mosquitoes were maintained at $28{ }^{\circ} \mathrm{C}$ and $80 \%$ relative humidity at a 12 -h light:12-h dark photoperiod. Adult mosquitoes were fed ad libitum 10\% glucose solution via impregnated cotton wool, while larvae were fed TetraMin fish flakes.

\section{Experimental drugs}

Based on their mechanism of action in humans, voriconazole, ritonavir, and cobicistat were classified as dual CYP/P-gp inhibitors [18, 19], cyclosporine A and elacridar were classified as P-gp-specific inhibitors [37], and rifampicin was classified as a dual CYP/P-gp inducer [38]. The choice of inhibitors and inducers used in the present study was based on their ability to act as substrates for the CYP CYP3A4, which is the major enzyme involved in ivermectin metabolism in humans [39].

Ivermectin, voriconazole, ritonavir, cobicistat, cyclosporine A, elacridar, and rifampicin were obtained from Sigma Aldrich (Spain). The active pharmacological ingredients were dissolved in dimethyl sulfoxide (DMSO) to prepare stock solutions for all compounds. Aliquots of the prepared solutions were frozen at $-20{ }^{\circ} \mathrm{C}$. For each experiment, the stock solutions were diluted in phosphate-buffered saline (PBS) to achieve the desired concentration.

\section{Membrane blood feeding}

For blood feeding, we used certified drug-free cattle blood defibrinated using the Reynold's method [40]. Briefly, after blood collection, the blood was gently shaken for 5-10 min in a $250 \mathrm{ml}$ glass bottle containing a copper wire arranged as an elongated spiral with two outer loops inside. The wire was then removed together with the fibrin that became bound to it [40]. The defibrinated blood was mixed with the drugs at appropriate concentrations to a total volume of $6 \mathrm{ml}$. In the case of the control group, the blood was mixed with PBS only.

Fifty 2-5-day-old blood-naïve female mosquitoes were transferred from stock cages to mosquito holding cups $\left(1000 \mathrm{~cm}^{3}\right)$ and starved of water and glucose for $6-8 \mathrm{~h}$ before blood feeding. The holding cups were covered by untreated net with a lateral aspiration hole covered with a double layer of dental dam. Blood feeding was done using an inverted cup technique [41]. The blood was placed on the bottom surface of a paper cup (500 ml capacity) and covered by a thinly stretched Parafilm membrane. The membrane was secured with masking tape, and the cup was inverted and filled with warm water $\left(\sim 38^{\circ} \mathrm{C}\right)$. The cups were then held over the netting material of the holding cups and mosquitoes were allowed to feed. Mosquito feeding was done in the dark for a period of 30-60 min. Visually unfed mosquitoes were removed and only fully engorged mosquitoes kept in the holding cages for follow-up and maintained at standard insectary conditions. Mortality was monitored every $24 \mathrm{~h}$ for 10 days by counting and removing dead mosquitoes. At least three replicates were performed for every drug or drug combination tested. The position of the cages was rotated daily.

\section{Data collection and statistical analysis}

Daily mortality data were entered in Excel spreadsheets. Survival Kaplan-Meier and Cox regression analyses were performed in Addinsoft XLSTAT ${ }^{\circledR}$ version 2018.5 software (New York, NY, USA) and GNU R (R Core Team [2020] R: A language and environment for statistical computing, version 3.6.3, R Foundation for Statistical Computing, Vienna, Austria, https://www.R-project. org). Comparisons of survival patterns were performed with the log-rank test using a $5 \%$ significance level. When the overall $p$ value was $<0.05$, pairwise comparisons were performed and Bonferroni correction used to correct for multiple comparisons.

\section{Results}

Ivermectin induces dose-dependent but delayed mortality in An. gambiae

We first determined the 10-day $\mathrm{LC}_{50}$ of ivermectin in our colony of An. gambiae s.s. The effect of ivermectin 


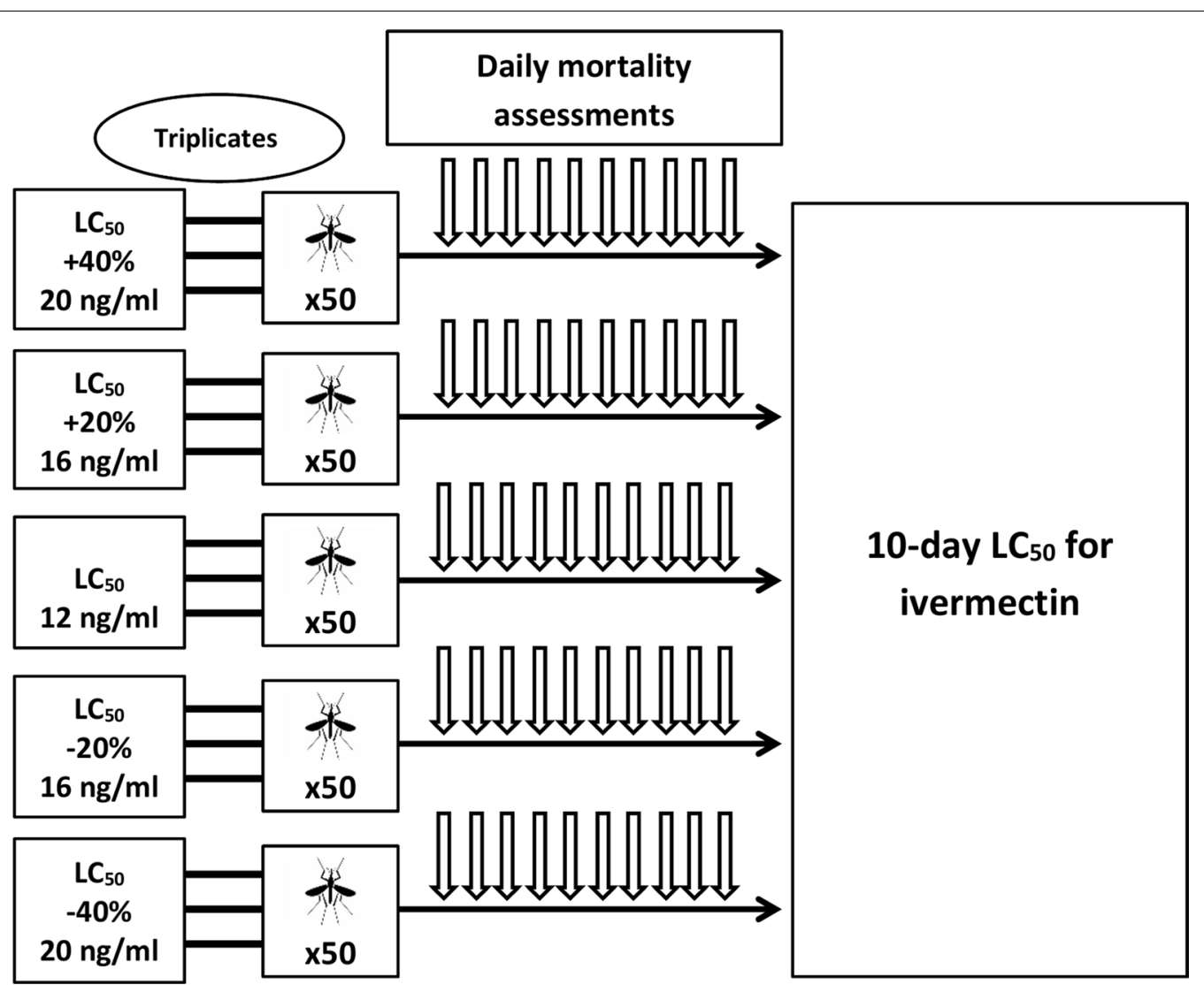

Fig. 1 Schematic representation of phase 1 experiments. Dose-finding study for ivermectin's 10-day insecticidal concentration $L C_{50}$ in our Anopheles gambiae s.s. colony. $\mathrm{LC}_{50}$ : insecticidal concentration 50

on mosquito survival was dose-dependent (Fig. 3). The $8 \mathrm{ng} / \mathrm{ml}$ concentration was the only one that resulted in approximately $50 \%$ mortality in 10 days, showing a mortality rate of $46.49 \%$ (Table 2). Therefore, this concentration of ivermectin was chosen for use in phase 2 experiments.

Mortality after ivermectin intake did not occur immediately and was negligible for the first 3 days. Instead, mosquito mortality occurred largely between days 4 and 6. Most of the mosquitoes surviving after day 6 remained alive until day 10 . These tendencies were observed across all the concentrations of ivermectin.

\section{Dual CYP/P-gp inhibitors have a synergistic effect on ivermectin-induced mosquito mortality}

Firstly, we excluded the possibility that any observed differences in mortality observed by combining ivermectin with a CYP/P-gp inhibitor were because of additive mortality caused by the CYP/P-gp inhibitors themselves.
No significant differences in mortality were observed between mosquitoes that consumed CYP/P-gp inhibitors alone and those that did not, irrespective of the dose used (Figs. 4a, c, 5a).

In contrast, differences in mortality were observed between mosquitoes that consumed ivermectin alone and those that consumed ivermectin combined with a dual CYP/P-gp inhibitor. Notably, experiments conducted with ritonavir and voriconazole showed enhanced ivermectin-induced mortality and dose-dependent synergism (Fig. 4b, d). Unlike voriconazole, the synergistic effect of ritonavir saturated at the second lowest concentration (concentration C). Additionally, when ivermectin was combined with ritonavir or voriconazole, an increase in the percentage of mortality and decrease in mean survival time of the mosquitoes were observed (Table 3). 


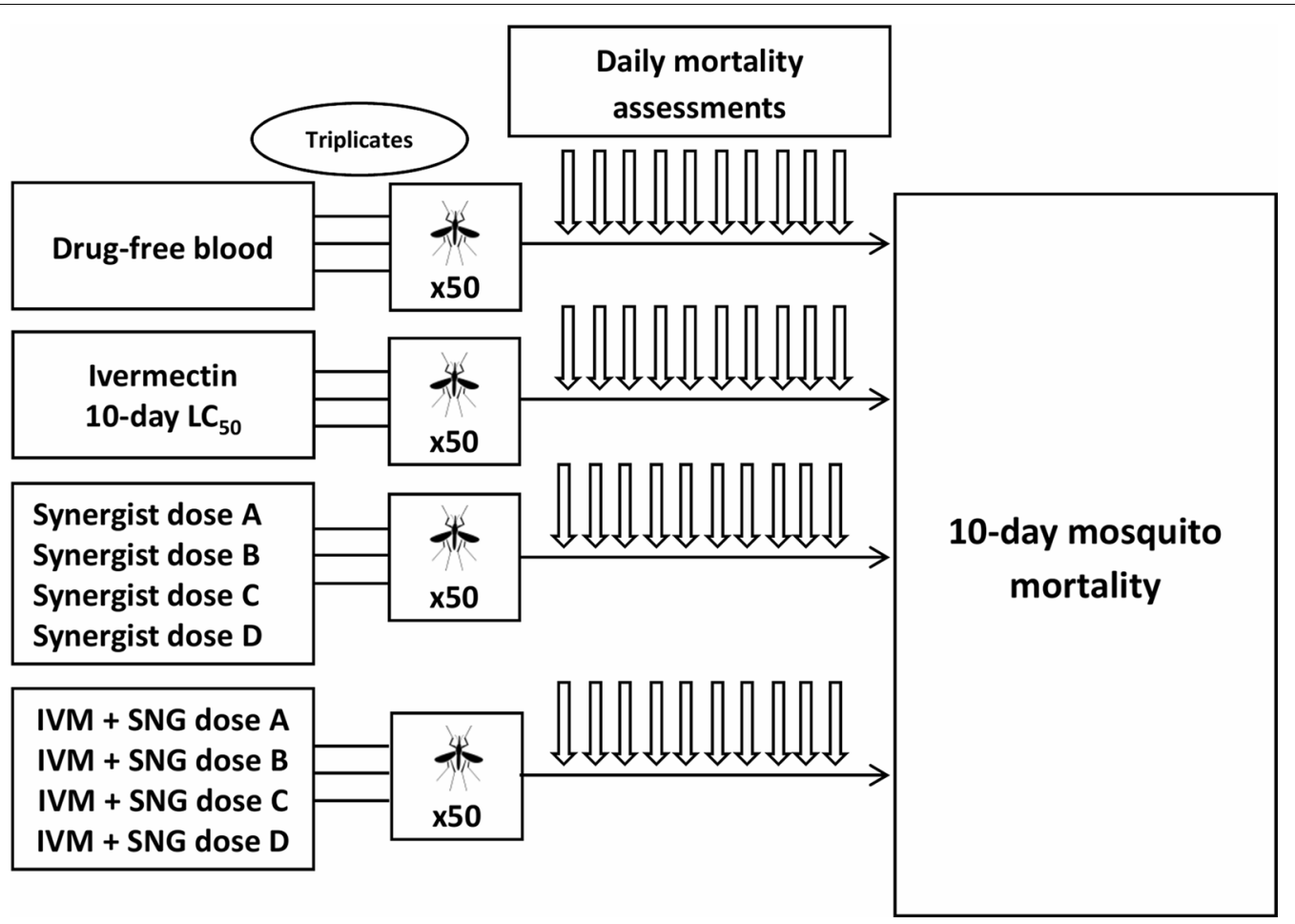

Fig. 2 Schematic representation of phase 2 experiments. Assessment of 10-day mosquito mortality after feeding on blood containing different concentrations of CYP/P-gp inhibitors and inducers, either alone or in combination with ivermectin at the 10-day $L_{50}$ dose determined in phase 1

Table 1 Synergists and corresponding doses used for the experiments

\begin{tabular}{|c|c|c|c|c|c|}
\hline Drug & Mechanism of action & $D_{A}\left(C_{\max }\right)(n g / m l)$ & $\begin{array}{l}D_{\mathrm{B}}\left(75 \% \text { of } C_{\max }\right) \\
(\mathrm{ng} / \mathrm{ml})\end{array}$ & $\begin{array}{l}\mathrm{D}_{\mathrm{C}}\left(50 \% \text { of } C_{\max }\right) \\
(\mathrm{ng} / \mathrm{ml})\end{array}$ & $\begin{array}{l}D_{\mathrm{D}}(25 \% \text { of } \\
\left.C_{\max }\right)(\mathrm{ng} / \\
\mathrm{ml})\end{array}$ \\
\hline Cobicistat [31] & Dual CYP/P-gp inhibitor ${ }^{a}$ & 990 & 742 & 495 & 247 \\
\hline Cyclosporine A [32] & Selective P-gp inhibitor ${ }^{b}$ & 1802 & 1351 & 901 & 450 \\
\hline Elacridar [33] & Selective P-gp inhibitor & 160 & 120 & 80 & 40 \\
\hline Rifampicin [34] & Dual CYP/P-gp inducer & 7000 & 5250 & 3500 & 1750 \\
\hline Ritonavir [35] & Dual CYP/P-gp inhibitor & 11,000 & 8250 & 5500 & 2750 \\
\hline Voriconazole [36] & Dual CYP/P-gp inhibitor & 3667 & 2750 & 1833 & 916 \\
\hline
\end{tabular}

a With effect markedly skewed towards CYP inhibition

${ }^{b}$ Negligible effect on CYPs

\section{Cobicistat, a strong antagonistic effect}

Cobicistat alone had no effect on mosquito mortality regardless of the concentration used (Fig. 5a). Despite being a dual CYP/P-gp inhibitor, cobicistat showed an antagonistic effect on ivermectin-induced mortality when combined with ivermectin. High cobicistat concentrations protected the mosquitoes from ivermectin toxicity, with a higher survival probability recorded in cobicistat combined with ivermectin than ivermectin alone (Fig. 5b). Additionally, when combined with ivermectin, cobicistat showed a lower percentage of mortality in comparison to ivermectin alone (Table 3 ).

Inhibition of P-gp alone does not affect ivermectin-induced mosquito mortality

After observing the effect of CYP/P-gp inhibition on ivermectin-induced mortality, we next assessed the effects of only inhibiting P-gp transporters. For this, we used elacridar and cyclosporine $\mathrm{A}$, which are predominantly P-gp inhibitors in vivo. Similarly to the dual CYP/P-gp 


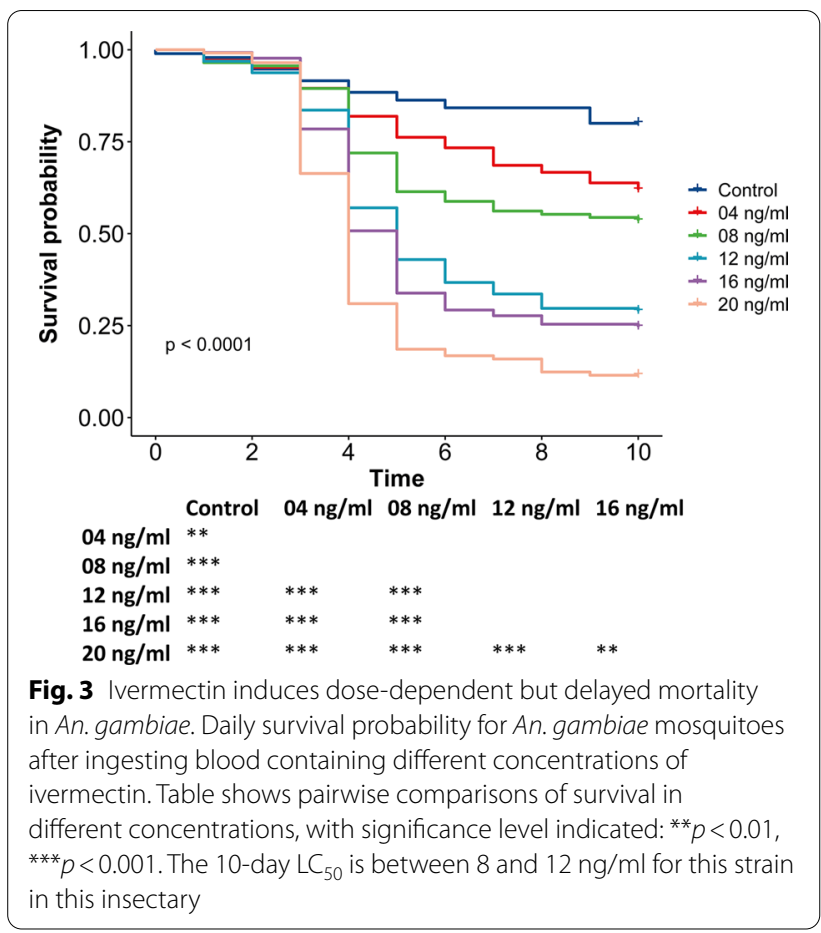

Table 2 Mosquito survival after ingestion of multiple concentrations of ivermectin

\begin{tabular}{lll}
\hline $\begin{array}{l}\text { Ivermectin concentration (ng/ } \\
\mathrm{ml})\end{array}$ & Mean survival time $(95 \% \mathrm{Cl})$ & $\begin{array}{l}\text { 10-day \% } \\
\text { mortality }\end{array}$ \\
\hline 0 (control) & $8.1(7.6-8.5)$ & 20 \\
4 & $8.1(7.5-8.6)$ & 38.1 \\
8 & $7.3(6.8-7.9)$ & 46.5 \\
12 & $6.0(5.5-6.5)$ & 71.1 \\
16 & $5.6(5.2-6.1)$ & 75.4 \\
20 & $4.5(4.1-4.9)$ & 88.5 \\
\hline
\end{tabular}

inhibitors, the selective P-gp inhibitors alone did not cause significant mortality at any dose tested (Fig. 6a, c).

Moreover, when combined with ivermectin, both elacridar and cyclosporine A showed no effect on ivermectin-induced mortality, suggesting that P-gp inhibitors do not synergize with ivermectin to increase mortality (Fig. 6b, d).

\section{Simultaneous induction of cytochrome P450 and P-gp may confer modest protection from ivermectin-induced mortality}

No significant difference was observed in the survival of mosquitoes feeding on rifampicin alone at the different doses tested (Fig. 7a).

However, when combined with ivermectin, rifampicin at a concentration close to $50 \%$ of its $C_{\max }$ for CYP $3 \mathrm{~A} 4$ induction showed a statistically significant difference from ivermectin alone; this was seen as antagonism by reducing the ivermectin-induced mortality (Table 3; Fig. 7b).

\section{Discussion}

Susceptibility to ivermectin has been shown to vary among mosquito species as well as among mosquito strains of the same species [27, 42, 43]. Depending on the time frame during which survival is monitored, An. gambiae have shown an $\mathrm{LC}_{50}$ of $19.8,15.9$, and $22.4 \mathrm{ng} / \mathrm{ml}$ when survival is monitored for 9,7 , and 5 days, respectively $[27,44,45]$. In our case, by monitoring survival for 10 days, we achieved $46.49 \%$ mortality with a concentration of $8 \mathrm{ng} / \mathrm{ml}$. Though the levels of ivermectin in the blood drop rapidly, a concentration above $8 \mathrm{ng} / \mathrm{ml}$ can be maintained for close to $36 \mathrm{~h}$ following an ivermectin dose in humans [14, 27]. Our results are aligned with those of Smit et al., in which even very low ivermectin concentrations increased mosquito mortality if the follow-up period encompassed the usual lifespan [46].

At the doses used, ivermectin-induced mortality in mosquitoes was not observed until 2-3 days postexposure. One potential explanation for this is the time taken for ivermectin to be absorbed from the midgut, as faster lethality onset has been observed when ivermectin is injected directly into the midgut than when it is taken as part of a blood meal [42]. The second plausible explanation for the delayed mortality is the involvement of ivermectin metabolites rather than the parent compound in causing mosquito mortality. There is accumulating evidence suggesting the involvement of ivermectin metabolites in mosquito mortality, though the specific metabolites are yet to be identified [46, 47]. However, even before the onset of lethality that is measurable with a 10-day follow up, ivermectin can potentially affect mosquito mortality in the wild due to its effects on locomotion [48]. Simultaneously, a reduction in locomotion ability would affect the vectorial capacity regardless of mosquito mortality, which could in turn further reduce malaria transmission.

Ivermectin-induced mortality is greatly dependent on attaining high systemic levels of ivermectin in the mosquito. The exposure to ivermectin is determined by the mosquito's detoxification capacity. Generally, in insects, detoxification processes involve metabolic enzymes such as CYP, esterase, and glutathione-S-transferases (GSTs) together with efflux pumps like the P-gp [49]. In this study, we investigated whether and how CYPs and P-gp transporters affected ivermectin-induced mortality in mosquitoes. Our results demonstrate that the simultaneous inhibition of CYPs and P-gp transporters 

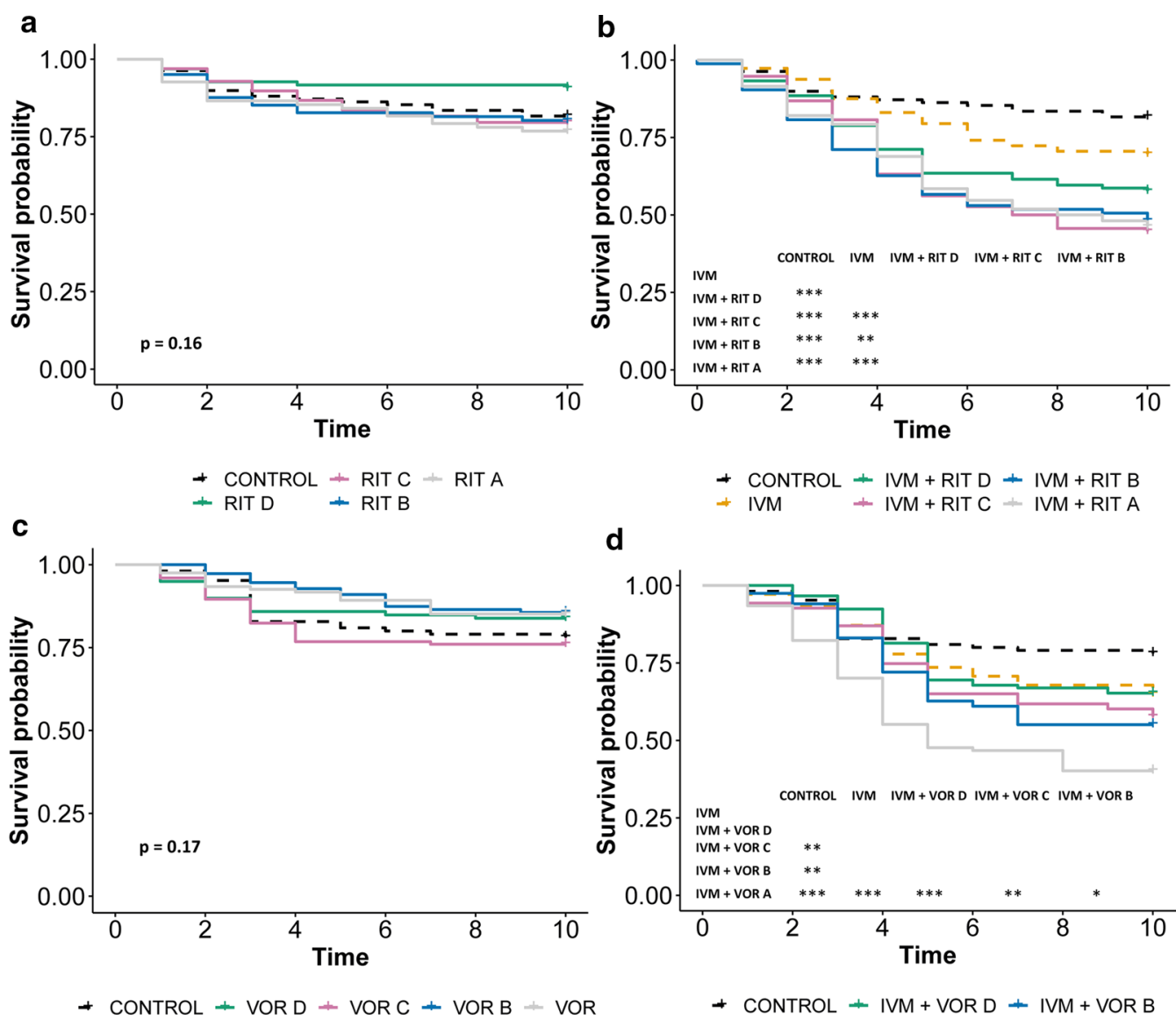

d

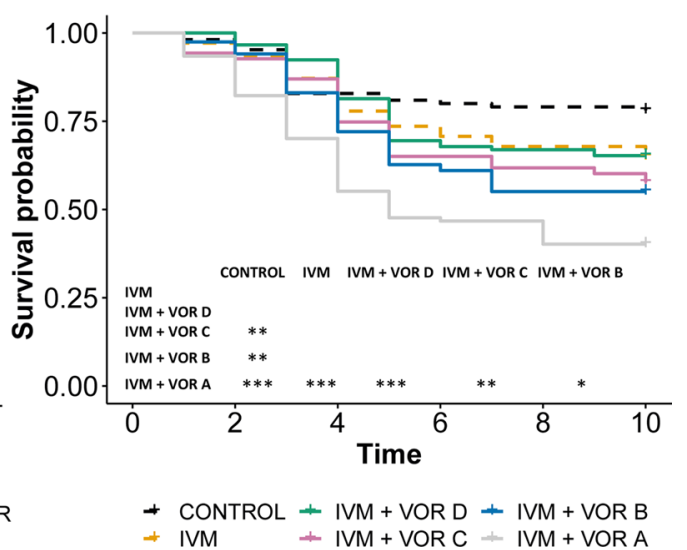

Fig. 4 Synergistic effect of dual CYP/ P-gp inhibitors on ivermectin-induced mosquito mortality. Daily survival probability of An. gambiae mosquitoes after imbibing blood containing (a) varied concentrations of ritonavir (RIT), (b) ivermectin (IVM) mixed with varied concentrations of ritonavir, (c) varied concentrations of voriconazole, and (d) ivermectin mixed with varied concentrations of voriconazole. When the overall $p$ value was $<0.05$, pairwise comparisons was performed and the significance level indicated: ${ }^{*} p<0.05,{ }^{* *} p<0.01,{ }^{* * *} p<0.001$. Concentration A $>B>C>D$
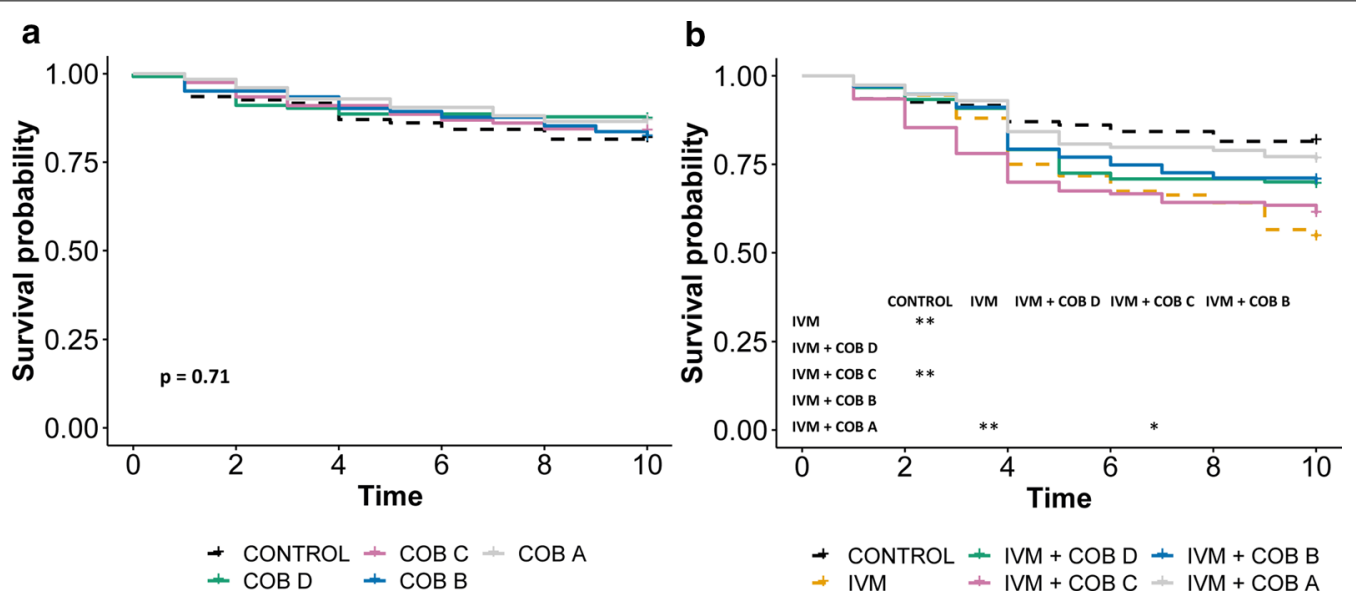

Fig. 5 Cobicistat appears to antagonize the ivermectin-induced mosquito mortality. Daily survival probability of An. Gambiae mosquitoes after ingesting blood containing (a) varied concentrations of cobicistat (COB), and (b) ivermectin (IVM) mixed with varied concentrations of cobicistat. When the overall $p$ value was $<0.05$, pairwise comparisons were performed and the significance level indicated: ${ }^{*} p<0.05,{ }^{* *} p<0.01,{ }^{* * *} p<0.001$. Concentration $\mathrm{A}>\mathrm{B}>\mathrm{C}>\mathrm{D}$ 
Table 3 Synergist drugs combined with ivermectin

\begin{tabular}{|c|c|c|c|}
\hline Drug & $\begin{array}{l}\text { Synergist } \\
\text { concentration (ng/ } \\
\mathrm{ml} \text { ) }\end{array}$ & $\begin{array}{l}\text { Mean survival } \\
\text { time }(95 \% \mathrm{CI})\end{array}$ & $\begin{array}{l}\text { 10-day \% } \\
\text { mortality }\end{array}$ \\
\hline \multicolumn{4}{|l|}{ Voriconazole } \\
\hline Control & & $8.5(8.0-9.1)$ & 21.9 \\
\hline Ivermectin & & $8.0(7.5-8.5)$ & 35.0 \\
\hline$I V M+V O R A$ & 3667 & $5.4(4.9-5.9)$ & 59.8 \\
\hline $\mathrm{IVM}+\mathrm{VOR} B$ & 2750 & $5.7(5.3-6.0)$ & 44.9 \\
\hline IVM+VORC & 1833 & $7.6(7.0-8.1)$ & 42.2 \\
\hline $\mathrm{IVM}+\mathrm{VOR} D$ & 916 & $7.4(6.9-7.8)$ & 34.7 \\
\hline \multicolumn{4}{|l|}{ Ritonavir } \\
\hline Control & & $8.0(7.5-8.4)$ & 18.3 \\
\hline Ivermectin & & $8.2(7.7-8.8)$ & 30.3 \\
\hline $\mathrm{IVM}+\mathrm{RIF} \mathrm{A}$ & 11,000 & $6.8(6.1-7.5)$ & 53.7 \\
\hline$I V M+R I F B$ & 8250 & $6.6(5.8-7.4)$ & 51.8 \\
\hline$I V M+R I F C$ & 5500 & $6.7(6.1-7.3)$ & 55.2 \\
\hline$I V M+R I F D$ & 2750 & $7.3(6.7-8.0)$ & 42.3 \\
\hline \multicolumn{4}{|l|}{ Cobicistat } \\
\hline Control & & $7.1(6.8-7.5)$ & 18.5 \\
\hline Ivermectin & & $7.8(7.1-8.4)$ & 45.6 \\
\hline $\mathrm{IVM}+\mathrm{COB} A$ & 990 & $8.6(8.1-9.1)$ & 23.6 \\
\hline $\mathrm{IVM}+\mathrm{COB} B$ & 742 & $8.2(7.8-8.7)$ & 29.6 \\
\hline $\mathrm{IVM}+\mathrm{COBC}$ & 495 & 7.5 (6.9-8.1) & 39.0 \\
\hline $\mathrm{IVM}+\mathrm{COB} \mathrm{D}$ & 247 & $8.1(7.6-8.6)$ & 30.8 \\
\hline \multicolumn{4}{|l|}{ Elacridar } \\
\hline Control & & $9.5(9.3-9.8)$ & 12.2 \\
\hline Ivermectin & & $7.1(6.5-7.6)$ & 54.6 \\
\hline $\mathrm{IVM}+\mathrm{ELA} A$ & 160 & $6.9(6.4-7.4)$ & 62.3 \\
\hline$I V M+E L A B$ & 120 & $7.3(6.8-7.8)$ & 49.6 \\
\hline IVM+ELAC & 80 & $6.5(6.1-7.0)$ & 51.4 \\
\hline IVM + ELA D & 40 & $7.0(6.5-7.4)$ & 56.1 \\
\hline \multicolumn{4}{|l|}{ Cyclosporine A } \\
\hline Control & & $9.0(8.3-9.6)$ & 15.6 \\
\hline Ivermectin & & $9.0(8.5-9.6)$ & 21.8 \\
\hline $\mathrm{IVM}+\mathrm{CYCA}$ & 1802 & $7.8(6.8-8.8)$ & 34.7 \\
\hline $\mathrm{IVM}+\mathrm{CYCB}$ & 1351 & $7.7(6.8-8.6)$ & 38.1 \\
\hline $\mathrm{IVM}+\mathrm{CYCC}$ & 901 & $7.7(7.0-8.4)$ & 20.9 \\
\hline $\mathrm{IVM}+\mathrm{CYCD}$ & 450 & $8.5(7.9-9.2)$ & 33.3 \\
\hline \multicolumn{4}{|l|}{ Rifampicin } \\
\hline Control & & $7.2(6.6-7.7)$ & 39.1 \\
\hline Ivermectin & & $6.7(6.0-7.5)$ & 53.4 \\
\hline $\mathrm{IVM}+\mathrm{RIF} A$ & 7000 & $6.9(6.2-7.6)$ & 49.5 \\
\hline IVM+RIF B & 5250 & $6.4(5.6-7.1)$ & 61.3 \\
\hline $\mathrm{IVM}+\mathrm{RIFC}$ & 3500 & $8.2(7.6-8.9)$ & 31.5 \\
\hline IVM + RIF D & 1750 & $6.4(5.8-7-0)$ & 66.3 \\
\hline
\end{tabular}

IVM ivermectin, VOR voriconazole, RIF ritonavir, COB cobicistat, ELA elacridar, CYC cyclosporine $\mathrm{A}$

enhances ivermectin-dependent mortality in a dosedependent manner, indicating synergism. However, this only happened selectively when ritonavir or voriconazole was used. Unexpectedly, the use of cobicistat, which is also a dual CYP/P-gp inhibitor, rendered some protection from ivermectin. Cobicistat is a structural analogue of ritonavir, but unlike ritonavir, which is known to inhibit and induce multiple CYPs, cobicistat more selectively inhibits CYP3A4 [50]. Though ritonavir and cobicistat are considered clinically equivalent, the small difference in ritonavir's ability to induce CYPs could result in differences in the drug-drug interaction $[51,52]$. The induction of CYPs could possibly lead to ivermectin metabolism, making ivermectin metabolites available.

Despite dual CYP/P-gp inhibitors showing an effect on ivermectin-induced mortality, P-gp selective inhibitors did not have a measurable effect. Taken together, our results suggest that detoxification mechanisms mediated by CYPs are more important in ivermectin detoxification. This is contrary to what has been reported in mosquito larval stages, where Buss et al. demonstrated that inhibition of P-gp using verapamil led to increased toxicity in Culex mosquitoes [23]. Collectively, both findings suggest heterogeneity in detoxification mechanisms in different stages of mosquito development. As a holometabolous insect, the changes between the immature stages (larvae and pupae) and the adult stage are characterized by differences in diet, habitat, morphology, physiology, and behavior. These differences could potentially lead to differences in evolution of protective mechanisms [53]. Larval stages are known to be more prone to developing insecticide resistance compared to the adult stage [53]. Whether the P-gp-mediated detoxification reported in Culex larvae is additive or alternative to CYP-mediated detoxification in larvae merits investigation. Larval habitats are often exposed to ivermectin through contamination of aquatic habitats with excreta from treated livestock. The stability of ivermectin in water for long periods increases the exposure of the larvae to ivermectin and could potentially accelerate the development of resistance [54]. It is important to understand the mechanisms behind larval resistance to ivermectin and whether they contribute to ivermectin resistance in adults.

Our results warrant the investigation of selective CYP inhibitors for the ability to synergize ivermectin-induced mortality, including molecules susceptible to administration by contact such as piperonyl butoxide (PBO). This will help answer the question of whether CYP inhibition independent of P-gp inhibition could still have practical implications. Notably, the current recommendation for tackling metabolic resistance to insecticides is the use of PBO [55]. $\mathrm{PBO}$, which is an inhibitor of CYP450 enzymes and is currently in use by incorporation into pyrethroid long-lasting insecticidal nets (LLINs), could also enhance ivermectininduced mortality. However, it must be first determined whether it synergizes ivermectin-induced mortality. 

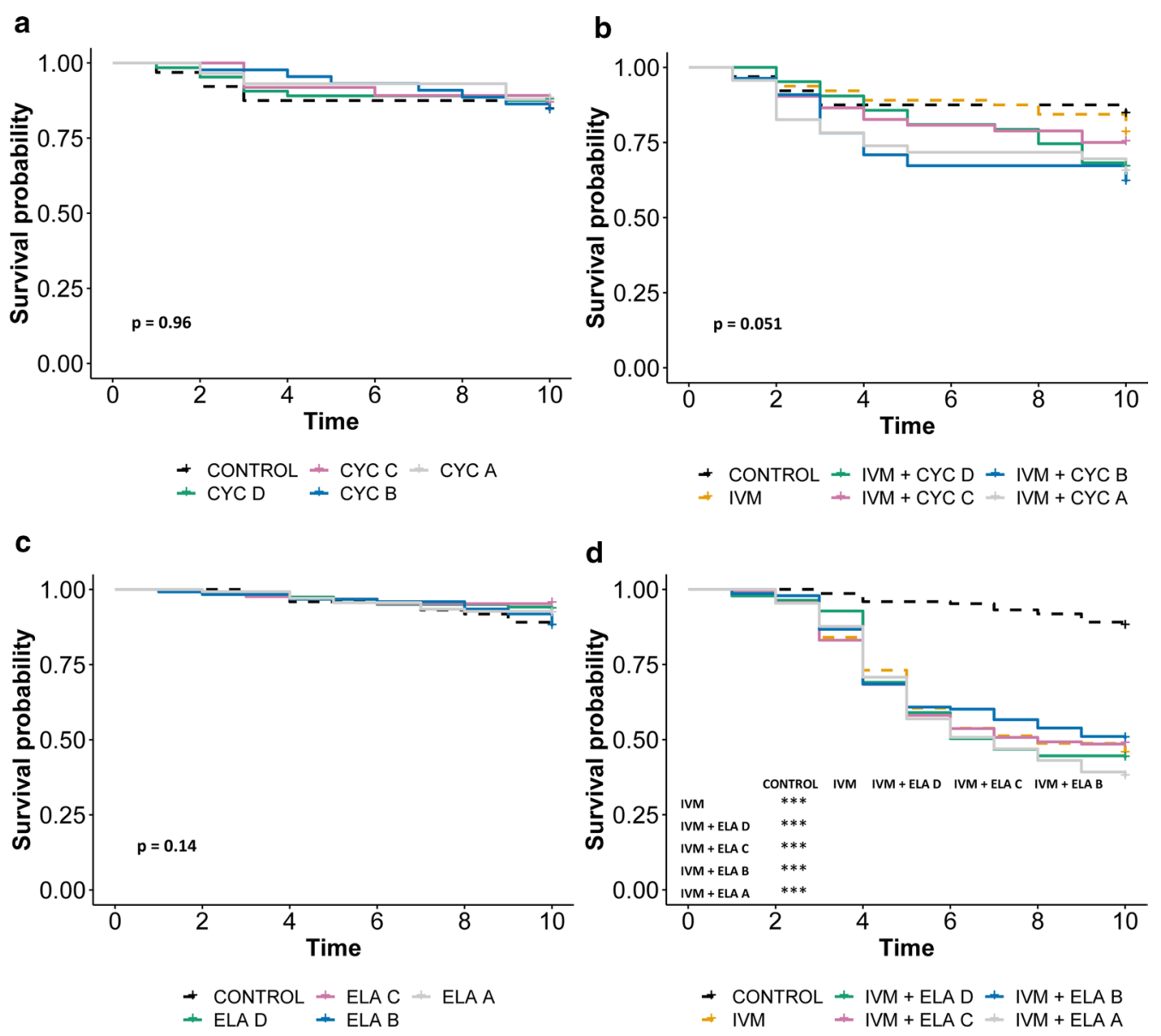

Fig. 6 Inhibition of P-gp alone does not affect ivermectin-induced mosquito mortality. Daily survival probability of An. Gambiae mosquitoes after ingesting blood containing (a) varied concentrations of cyclosporine A (CYC), (b) ivermectin (IVM) mixed with varied concentrations of cyclosporine A, (c) varied concentrations of elacridar (ELA), and (d) ivermectin mixed with varied concentrations of elacridar. When the overall $p$ value was $<0.05$, pairwise comparisons were performed and the significance level indicated: ${ }^{*} p<0.05,{ }^{* *} p<0.01,{ }^{* * *} p<0.001$. Concentration A $>B>C>D$
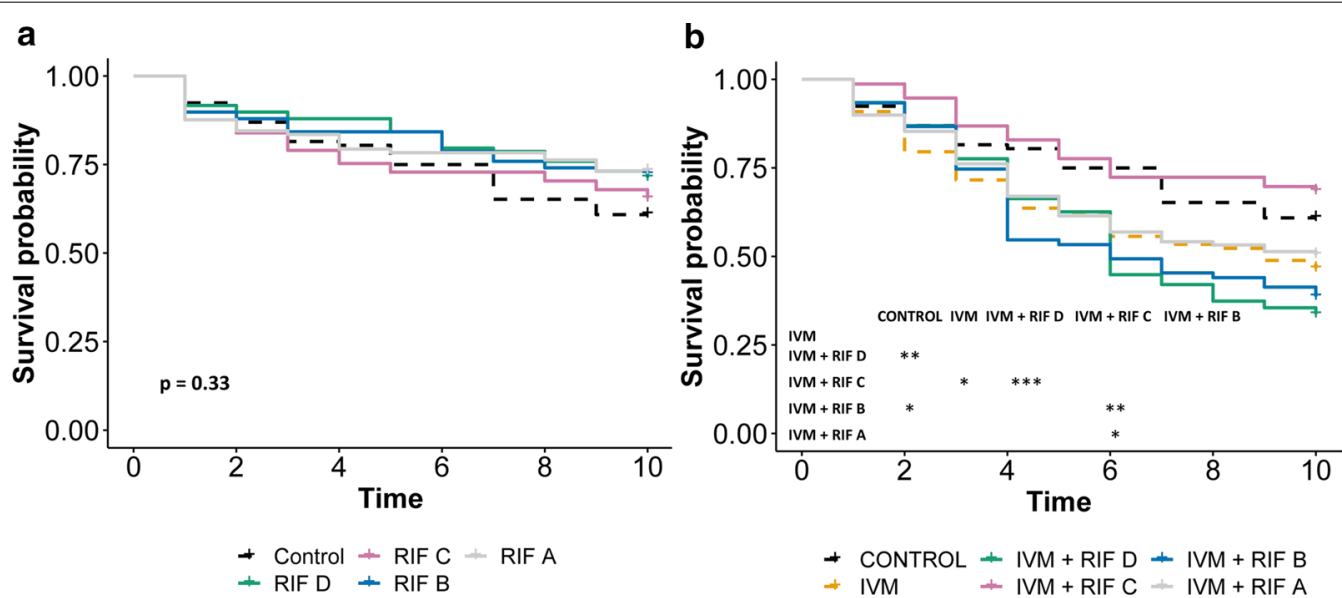

Fig. 7 Simultaneous induction of cytochrome P450 and P-gp transporters may confer modest protection from ivermectin-induced mortality. Daily survival probability of An. Gambiae mosquitoes after ingesting blood containing (a) varied concentrations of rifampicin (RIF) and (b) ivermectin (IVM) mixed with varied concentrations of rifampicin. When the overall $p$ value was $<0.05$, pairwise comparisons were performed and the significance level indicated: ${ }^{*} p<0.05,{ }^{* *} p<0.01,{ }^{* * *} p<0.001$. Concentration $A>B>C>D$ 
Limitations of this work include the following: (1) The ivermectin concentration used for the combined experiments did not quite yield $50 \%$ mortality in 10 days, but only $46 \%$. (2) The initial solutions of synergistic molecules were prepared using small quantities of DMSO and then diluted in PBS to the desired concentration. The control solution did not contain DMSO, as previous experiments have shown that it does not alter mosquito survival at low concentrations [27]. (3) It is still unclear whether the finding that only one rifampicin concentration demonstrated an interaction with ivermectin is an artifact or an effect limited to a certain concentration range, and (4) it is also unclear why ritonavir and cobicistat interacted in such different ways with ivermectin when both molecules strongly inhibit CYP 3A4. Still, the involvement of CYPs in ivermectin metabolism could potentially lead to crossresistance between ivermectin and current insecticides used in vector control. Deus et al. previously demonstrated that pyrethroid-resistant Ae. aegypti have higher tolerance to ivermectin [43]. This could potentially affect ivermectin susceptibility in mosquito populations already showing metabolic resistance to insecticides, and highlights the need to investigate the impact of insecticide resistance on susceptibility to ivermectin.

Nevertheless, ivermectin remains a good alternative for mosquito populations whose mode of resistance is via point mutations, since this resistance mechanism is not analogous between the current insecticides and ivermectin [56]. While resistance to insecticides is caused by mutations in the sodium channel, acetylcholinesterase, or gamma-aminobutyric acid (GABA) receptor genes, ivermectin resistance in other arthropods is associated with mutations involving the glutamate-gated chloride channels (GluCls) $[57,58]$.

In the case where ivermectin is to be used in mosquito populations with metabolic resistance to the current insecticides, our study provides insights into the possibility of ivermectin cross-resistance with other insecticides. Our results suggest that detoxification mechanisms mediated by CYPs are more important in ivermectin resistance than detoxification mediated by efflux pumps.

\section{Acknowledgements}

Not applicable.

\section{Authors' contributions \\ Conceptualization: CCh. Data curation: CCh, PN, MM. Formal analysis: CCh, PN, CK. Funding acquisition: CCh. Investigation: PN, TBD, MM, MGW, MMt. Method- ology: CCh, MM, MGW. Supervision: MM, CCh. Writing-original draft: PN, CK, CCh. Writing - review and editing: all authors. All authors read and approved the final manuscript.}

\section{Funding}

This study was supported by an early career Grant award to CCh from Thrasher Research Fund. PN, CK, MM, and CCh received salary support from Unitaid through the BOHEMIA Grant to ISGlobal. ISGlobal acknowledges support from the Spanish Ministry of Science and Innovation through the "Centro de Excelencia Severo Ochoa 2019-2023" Program (CEX2018-000806-S), and support from the Generalitat de Catalunya through the CERCA Program. The funding source had no role on the design, analysis, or decision to publish the results of this study.

\section{Availability of data and materials}

All study data are contained within this manuscript.

\section{Declarations}

\section{Ethics approval and consent to participate}

This work does not involve humans or vertebrates and did not require IRB approval.

\section{Consent for publication}

Not applicable.

\section{Competing interests}

The authors declare that they have no competing interests.

\section{Author details}

${ }^{1}$ ISGlobal, Hospital Clínic-Universitat de Barcelona, Rosello 132, 5a 2a, 08036 Barcelona, Spain. ${ }^{2}$ Centro de Investigação em Saúde de Manhiça, 1929 Maputo, Mozambique. ${ }^{3}$ Wellcome Sanger Institute, Wellcome Genome Campus, Hinxton, Cambridge CB10 91SA, UK. ${ }^{4}$ Department of Biosciences, KEMRI Wellcome Trust Research Programme, Kilifi 230-80108, Kenya. ${ }^{5}$ Division of Clinical Pharmacology and Toxicology, Department of Biomedicine, University and University Hospital Basel, 4056 Basel, Switzerland. ${ }^{6}$ Division of Clinical Pharmacology and Toxicology, Department of Pharmaceutical Sciences, University of Basel, 4056 Basel, Switzerland. ${ }^{7}$ Division of Clinical Pharmacology and Toxicology, Department of Internal Medicine, University Hospital Bern 3010 Bern, Switzerland. ${ }^{8}$ Nuffield Department of Medicine, Centre for Tropical Medicine and Global Health, University of Oxford, Oxford, UK. ${ }^{9}$ Ifakara Health Institute, Ifakara 67501, United Republic of Tanzania. ${ }^{10}$ Facultad de Medicina, Universidad de Navarra, 31008 Pamplona, Spain.

Received: 1 December 2020 Accepted: 7 March 2021

Published online: 20 March 2021

References

1. WHO. World Malaria Report 2019. Geneva. World Malaria Report (2019).

2. Chan, M. Ten years in public health 2007-2017. World Health Organization (2017)

3. Bhatt $\mathrm{S}$ et al. The effect of malaria control on Plasmodium falciparum in Africa between 2000 and 2015. Nature 2015;526.

4. Mwangangi JM et al. Shifts in malaria vector species composition and transmission dynamics along the Kenyan coast over the past 20 years. 2013;12.

5. Russell TL et al. Increased proportions of outdoor feeding among residual malaria vector populations following increased use of insecticide-treated nets in rural Tanzania. 2011;10

6. Ranson H, Lissenden N. Insecticide resistance in African Anopheles mosquitoes: a worsening situation that needs urgent action to maintain malaria control. Trends Parasitol. 2016. https://doi.org/10.1016/j.pt.2015. 11.010 .

7. Sougoufara S, Ottih EC, Tripet F. The need for new vector control approaches targeting outdoor biting Anopheline malaria vector communities. Parasit Vectors. 2020. https://doi.org/10.1186/s13071-020-04170-7.

8. WHO. Ivermectin for malaria transmission control. World Health Organisation (2016).

9. Billingsley $\mathrm{P}$, et al. A roadmap for the development of ivermectin as a complementary malaria vector control tool. Am J Trop Med Hyg. 2020. https://doi.org/10.4269/ajtmh.19-0620.

10. Alout $\mathrm{H}$, et al. Evaluation of ivermectin mass drug administration for malaria transmission control across different West African environments. Malar J. 2014. https://doi.org/10.1186/1475-2875-13-417. 
11. Chaccour C, Lines J, Whitty CJM. Effect of ivermectin on Anopheles gambiae mosquitoes fed on humans: the potential of oral insecticides in malaria control. J Infect Dis. 2010. https://doi.org/10.1086/653208.

12. Hotez PJ, Molyneux DH. Tropical anemia: one of Africa's great killers and a rationale for linking malaria and neglected tropical disease control to achieve a common goal. PLoS Negl Trop Dis. 2008. https://doi.org/10. 1371/journal.pntd.0000270.

13. Gonzalez P, Gonzalez FA, Ueno K. Ivermectin in human medicine an overview of the current status of its clinical applications. Curr Pharm Biotechnol. 2012. https://doi.org/10.2174/138920112800399248.

14. Chaccour C, Hammann F, Rabinovich NR. Ivermectin to reduce malaria transmission I. Pharmacokinetic and pharmacodynamic considerations regarding efficacy and safety. Malar J. 2017. https://doi.org/10.1186/ s12936-017-1801-4.

15. Bass $C$, Jones $C M$. Mosquitoes boost body armor to resist insecticide attack. Proc Natl Acad Sci. 2016;113:9145-7.

16. Sampaio VS, et al. Filling gaps on ivermectin knowledge: effects on the survival and reproduction of Anopheles aquasalis, a Latin American malaria vector. Malar J. 2016;15:491.

17. Mekuriaw $W$, et al. The effect of ivermectin ${ }^{\circledR}$ on fertility, fecundity and mortality of Anopheles arabiensis fed on treated men in Ethiopia. Malar J. 2019;18:357.

18. Amanzougaghene $\mathrm{N}$, et al. Mutations in $\mathrm{GluCl}$ associated with field ivermectin-resistant head lice from Senegal. Int J Antimicrob Agents. 2018;52:593-8.

19. Lawrence J, Sodahlon YK, Ogoussan KT, Hopkins AD. Growth, challenges, and solutions over 25 years of mectizan and the impact on onchocerciasis control. PLoS Negl Trop Dis. 2015;9:0003507.

20. Dermauw W, Van Leeuwen T. The ABC gene family in arthropods: comparative genomics and role in insecticide transport and resistance. Insect Biochem Mol Biol. 2014. https://doi.org/10.1016/j.ibmb.2013.11.001.

21. Pignatelli $P$, et al. The Anopheles gambiae ATP-binding cassette transporter family: phylogenetic analysis and tissue localization provide clues on function and role in insecticide resistance. Insect Mol Biol. 2018;27:110-22.

22. Liu N. Insecticide resistance in mosquitoes: impact, mechanisms, and research directions. Annu Rev Entomol. 2015;60:537-59.

23. Buss DS, McCaffery AR, Callaghan A. Evidence for p-glycoprotein modification of insecticide toxicity in mosquitoes of the Culex pipiens complex. Med Vet Entomol. 2002. https://doi.org/10.1046/j.1365-2915.2002. 00365.x.

24. Pohl PC, et al. ABC transporter efflux pumps: a defense mechanism against ivermectin in Rhipicephalus (Boophilus) microplus. Int J Parasitol. 2011. https://doi.org/10.1016/j.ijpara.2011.08.004.

25. Le Gall VL, Klafke GM, Torres TT. Detoxification mechanisms involved in ivermectin resistance in the cattle tick, Rhipicephalus (Boophilus) microplus. Sci Rep. 2018. https://doi.org/10.1038/s41598-018-30907-7.

26. Blanford J, et al. Implications of temperature variation for malaria parasite development across Africa. Sci Rep. 2013:3:1300.

27. Kobylinski KC, et al. The effect of oral anthelmintics on the survivorship and re-feeding frequency of anthropophilic mosquito disease vectors. Acta Trop. 2010. https://doi.org/10.1016/j.actatropica.2010.06.001.

28. Hayes RJ, Bennett S. Simple sample size calculation for cluster-randomized trials. Int J Epidemiol. 1999;28:319-26.

29. Ouédraogo AL, et al. Efficacy and safety of the mosquitocidal drug ivermectin to prevent malaria transmission after treatment: a doubleblind, randomized, clinical trial. Clin Infect Dis Off Publ Infect Dis Soc Am. 2015;60:357-65.

30. Gangnon RE. Sample-size formula for clustered survival data using weighted log-rank statistics. Biometrika. 2004;91:263-75.

31. Tybost (cobicistat) dosing, indications, interactions, adverse effects, and more. https://reference.medscape.com/drug/tybost-cobicistat-999845\# 10.

32. FDA. NEORAL ${ }^{\circledR}$ Soft Gelatin Capsules (cyclosporine capsules, USP) MODIFIED NEORAL ${ }^{\circledR}$ Oral Solution (cyclosporine oral solution, USP) MODIFIED (2015).

33. Kuppens IELM, et al. A phase I, randomized, open-label, parallel-cohort, dose-finding study of elacridar (GF120918) and oral topotecan in cance patients. Clin Cancer Res. 2007;13:3276-85.

34. FDA. RIFADIN ${ }^{\circledR}$ (rifampin capsules USP) and RIFADIN ${ }^{\circledR}$ IV (rifampin for injection USP).
35. Hsu A Granneman GR, Bertz RJ Ritonavir Clinical pharmacokinetics and interactions with other anti-HIV agents. Clin Pharmacokinet. 1998:35:275-91.

36. FDA. Highlights of prescribing information. VFEND ${ }^{\circledR}$ (voriconazole) tablets for oral use VFEND ${ }^{\circledR}$ (voriconazole) for oral suspension VFEND ${ }^{\circledR}$ (voriconazole) for injection, for intravenous use.

37. Rautio J, et al. In vitro P-glycoprotein inhibition assays for assessment of clinical drug interaction potential of new drug candidates: a recommendation for probe substrates. Drug Metab Dispos. 2006. https://doi.org/10. 1124/dmd.105.008615.

38. Niemi M, Backman JT, Fromm MF, Neuvonen PJ, Kivistö KT. Pharmacokinetic interactions with rifampicin: clinical relevance. Clin Pharmacokinet. 2003. https://doi.org/10.2165/00003088-200342090-00003.

39. Zeng Z, Andrew NW, Arison BH, Luffer-Atlas D, Wang RW. Identification of cytochrome P4503A4 as the major enzyme responsible for the metabolism of ivermectin by human liver microsomes. Xenobiotica. 1998. https://doi.org/10.1080/004982598239597.

40. Reynolds FHK. A simple method for the defibrination of blood. J Am Med Assoc. 1919. https://doi.org/10.1001/jama.1919.26110150003011c.

41. Siria DJ, et al. Evaluation of a simple polytetrafluoroethylene (PTFE)-based membrane for blood-feeding of malaria and dengue fever vectors in the laboratory. Parasit Vectors. 2018;11:236.

42. Dreyer SM, Morin KJ, Vaughan JA. Differential susceptibilities of Anopheles albimanus and Anopheles stephensi mosquitoes to ivermectin. Malar J. 2018. https://doi.org/10.1186/s12936-018-2296-3.

43. Deus KM, Saavedra-Rodriguez K, Butters MP, Black WC IV, Foy BD. The effect of ivermectin in seven strains of aedes aegypti (Diptera: Culicidae) including a genetically diverse laboratory strain and three permethrin resistant strains. J Med Entomol. 2012. https://doi.org/10.1603/ME11164.

44. Fritz ML, et al. Toxicity of bloodmeals from ivermectin-treated cattle to Anopheles gambiae s.I. Ann Trop Med Parasitol. 2009. https://doi.org/10. 1179/000349809X12459740922138.

45. Kobylinski KC, Foy BD, Richardson JH. Ivermectin inhibits the sporogony of Plasmodium falciparum in Anopheles gambiae. Malar J. 2012. https:// doi.org/10.1186/1475-2875-11-381.

46. Smit MR, et al. Safety and mosquitocidal efficacy of high-dose ivermectin when co-administered with dihydroartemisinin-piperaquine in Kenyan adults with uncomplicated malaria (IVERMAL): a randomised, doubleblind, placebo-controlled trial. Lancet Infect Dis. 2018;18:615-26.

47. Kobylinski KC, et al. Safety, pharmacokinetics, and mosquito-lethal effects of ivermectin in combination with dihydroartemisinin-piperaquine and primaquine in healthy adult Thai subjects. Clin Pharmacol Ther. 2020;107:1221-30.

48. Sampaio VDS, et al. What does not kill it makes it weaker: effects of sub-lethal concentrations of ivermectin on the locomotor activity of Anopheles aquasalis. Parasit Vectors. 2017. https://doi.org/10.1186/ s13071-017-2563-0.

49. Panini M, Manicardi GC, Moores GD, Mazzoni E. An overview of the main pathways of metabolic resistance in insects. Invertebr Surviv J. (2016).

50. Marzolini C, Gibbons S, Khoo S, Back D. Cobicistat versus ritonavir boosting and differences in the drug-drug interaction profiles with comedications. J Antimicrob Chemother. 2016. https://doi.org/10.1093/jac/ dkw032.

51. Tseng A, Hughes CA, Wu J, Seet J, Phillips EJ. Cobicistat versus ritonavir: similar pharmacokinetic enhancers but some important differences. Ann Pharmacother. 2017. https://doi.org/10.1177/1060028017717018.

52. von Hentig N. Clinical use of cobicistat as a pharmacoenhancer of human immunodeficiency virus therapy. HIV/AIDS Res Palliative Care. 2015. https://doi.org/10.2147/HIV.S70836.

53. Koella JC, Lynch PA, Thomas MB, Read AF. Towards evolution-proof malaria control with insecticides. Evol Appl. 2009. https://doi.org/10. 1111/j.1752-4571.2009.00072.x.

54. Liebig M, et al. Environmental risk assessment of ivermectin: a case study. Integr Environ Assess Manag. 2010. https://doi.org/10.1002/ieam.96.

55. WHO. Conditions for deployment of mosquito nets treated with a pyrethroid and piperonyl butoxide. Glob Malar Programme World Health Organ. 2017. https://doi.org/10.1007/s40122-017-0070-9.

56. Alout H, Foy BD. Ivermectin: a complimentary weapon against the spread of malaria? Expert Rev Anti Infect Ther. 2017;15:231-40. 
57. Wang $X$, et al. A point mutation in the glutamate-gated chloride channel of Plutella xylostella is associated with resistance to abamectin. Insect Mol Biol. 2016. https://doi.org/10.1111/imb.12204.

58. Hemingway J, Hawkes NJ, McCarroll L, Ranson H. The molecular basis of insecticide resistance in mosquitoes. Insect Biochem Mol Biol. 2004. https://doi.org/10.1016/j.ibmb.2004.03.018.

\section{Publisher's Note}

Springer Nature remains neutral with regard to jurisdictional claims in published maps and institutional affiliations.
Ready to submit your research? Choose BMC and benefit from:

- fast, convenient online submission

- thorough peer review by experienced researchers in your field

- rapid publication on acceptance

- support for research data, including large and complex data types

- gold Open Access which fosters wider collaboration and increased citations

- maximum visibility for your research: over $100 \mathrm{M}$ website views per year

At BMC, research is always in progress.

Learn more biomedcentral.com/submissions 